\title{
Error Propagation in Extrapolated Nuclear Mass Predictions*
}

\author{
E. Comay and I. Kelson \\ Department of Physics and Astronomy, Tel Aviv University Tel Aviv, Israel
}

Received June 3, 1982; revised version October 22, 1982

\begin{abstract}
The properties of the error of the nuclear masses calculated from the transverse mass relations are analysed. The work assumes that the calculated errors of the nuclei whose masses are known experimentally behave as a sample selected randomly from a normal population having a zero mean and a standard deviation $\sigma$. It is found that the errors of the calculated masses of nuclei far from the line of beta-stability behave as $c_{1} d^{3 / 2}$ where $c_{1}$ is a constant and $d$ is the distance of the nucleus from the line of betastability. It is shown also that the errors related to the calculated mass differences behave as $c_{2} d^{\frac{1}{2}}$ where $c_{2}$ is another constant.
\end{abstract}

\section{Introduction}

A nuclear mass table with estimates of the errors of the calculated masses has been published [1]. In what follows we shall mainly try to analyse the general behavior of these errors. The analysis will obviously not be based upon theoretical considerations, since we are far from having a solid base for a theory of nuclear interactions. Instead, we shall use here certain assumptions and statistical arguments in order to derive some rules and conclusions about the behavior of the calculated errors.

There are two different methods of using the transverse mass relations for deriving a table of calculated nuclear masses:

A) The traditional method $[2,3]$ treats the transverse mass relations

$$
\begin{aligned}
& M(Z, N+1)+M(Z-1, N)+M(Z+1, N-1) \\
& -M(Z, N-1)-M(Z+1, N)-M(Z-1, N+1)=0
\end{aligned}
$$

as a homogeneous partial difference equation whose general solution is

$M(Z, N)=F(Z)+G(N)+H(Z+N)$.

The values of the functions $F, G$ and $H$ on the right hand side of (2) are obtained from a fit to the

* Work supported by the United States - Israel Binational Science Foundation experimental masses. Later, these values are used in the process of calculating a nuclear mass table. The fit is obtained from the solution of one set of conjugate equations. Due to this fact, we shall call this method the global method.

B) In this method [1] the mass relations are applied directly to a subset of the experimental masses. Using different subsets of the experimental masses, one can have different calculated values for the mass of a nucleus. The mean of these values is taken to be the calculated mass of the nucleus, and the standard error of these values is taken as an estimate of the error related to the calculated mean. Since this method uses expression (1), which involves only the masses of the nuclei in the vicinity of the nucleus whose mass is calculated, we shall call it the local method.

If the transverse mass relations were accurate, then the results of the two methods would be the same (and the errors of the calculation would be zero). Since this is not the case, differences between the results obtained by the two method arise. These differences stem from the fact that in the global method all experimental masses influence the calculation of each mass via the conjugate set of equations. In the local method, on the other hand, the calculation of $M\left(Z_{0}, N_{0}\right)$ does not depend on the experimental mass of a nucleus $M(Z, N)$ if the follow- 
ing relation holds: $\left(Z-Z_{0}\right)\left(N-N_{0}\right)>0$. Other differences between the two methods are related to technical properties of the work, e.g., assigning different weights to the experimental masses.

The side by side publication of the calculated masses [4] using nine different methods enables one to compare the differences between them. It appears, in spite of what was written above, that the global and the local methods of applying the transverse mass relations yield very similar results.

\section{Analysis of Errors in Predicted Masses}

We turn now to the analysis of the errors of the calculated masses obtained by the local method. To this end we shall describe here some details of the work.

A special subset of experimental masses, from which a mass table can be easily calculated will be called a skeleton. A skeleton has the following properties:

A) A skeleton consists of experimental masses of nuclei in the interval $\left(A_{0}, A_{1}\right)$.

$B)$ Two neighboring nuclei $(A, Z)$ and $(A, Z+1)$ belong to the skeleton for every $A$ in the interval $\left(A_{0}, A_{1}\right)$.

$C)$ If $A$ and $(A+1)$ are in the interval $\left(A_{0}, A_{1}\right)$ and the nuclei $(A, Z)$ and $(A, Z+1)$ belong to the skeleton, then the nucleus $(A+1, Z+1)$ belongs to the skeleton, too.

An example of a skeleton can be seen in Fig. 1.

We define the length of the skeleton as the number of pairs of nuclei belonging to it. We define also the range of a skeleton as the set of nuclei belonging to it, together with the nuclei whose masses can be calculated from the masses of the nuclei in the skeleton, using the transverse mass relations.

We shall show that a nucleus $\left(Z_{0}, N_{0}\right)$ is in the range of the skeleton if, and only if, a nucleus $\left(Z_{0}, N\right)$ and a nucleus $\left(Z, N_{0}\right)$ belong to the skeleton.

We use induction on the length of the skeleton. When the length is 1 , there is nothing to prove. Assume that the statement holds for all skeletons whose length is $K$, and let us take a skeleton whose length is $(K+1)$. Assume that the last pair is obtained by adding one neutron to the pair of nuclei having the highest value of $A$ in a skeleton whose length is $K$. (The proof is similar if one proton is added). Let us examine Fig. 1. Using the induction assumption, we can calculate the masses of the nuclei denoted by $x$. We can apply the transverse mass relation and calculate the mass of the nucleus denoted by 1 . This process can be continued until the masses of all the nuclei denoted by numbers are

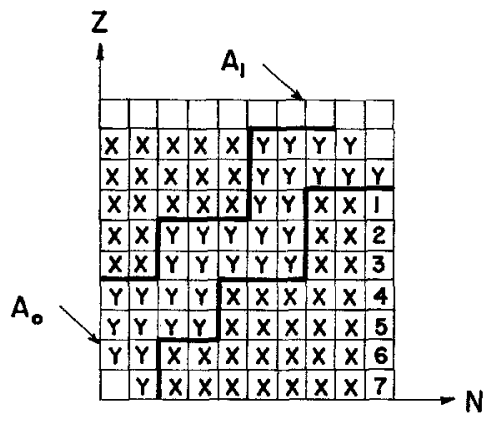

Fig. 1. The skeleton consists of the nuclei denoted by $Y$. The length of the skeleton is $\left(A_{1}-A_{0}+1\right)$. The range of the skeleton consists of all the nuclei denoted by letters or digits

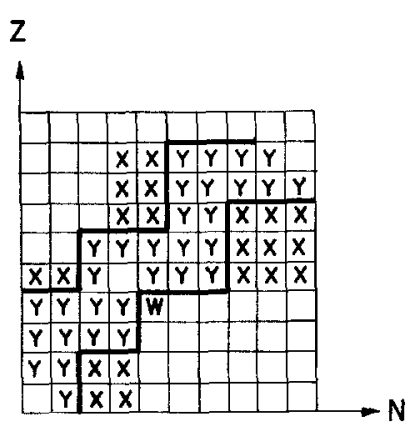

Fig. 2. A set of nuclei obtained from the skeleton of Fig. 1 after deleting one nucleus. Only nuclei denoted by $x$ and $w$ can be calculated, using the transverse mass relations

calculated. Moreover, nothing else can be calculated.

We shall show now that the skeleton is a minimal set. (i.e. there is no proper subset of the skeleton having the same range). Here, too, we shall use induction on the length of the skeleton. The statement is correct for a skeleton of length 1 . Assume that it is correct for a skeleton whose length is $K$. Let us take a skeleton whose length is $(K+1)$. If the statement is incorrect, then we have a set $S$ of nuclei which is obtained from a skeleton after deleting one nucleus form it (see Fig. 2). Let $A=A_{d}$ be the number of nucleons in the deleted nucleus. In $S$ we generally have two subskeletons, having $A<A_{d}$ and $A>A_{d}$, respectively. If $A_{d}=A_{\max }$ or $A_{d}=A_{\min }$, then we have only one subskeleton but the following demonstration is analogous. Using what was shown above, we can calculate the masses of the nuclei denoted by $x$ in Fig. 2. We can also calculate the mass of the nucleus denoted by $w$, but no more. This is a contradiction to the assumption that $S$ has the same range as the entire skeleton.

We shall use these properties in our attempt to make a prediction on the behavior of the errors of the calculated masses.

$A$ regular skeleton satisfies the following requirements: 


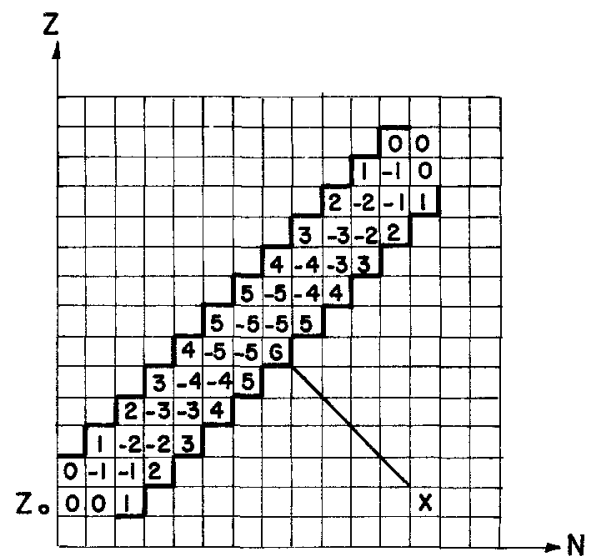

(1)

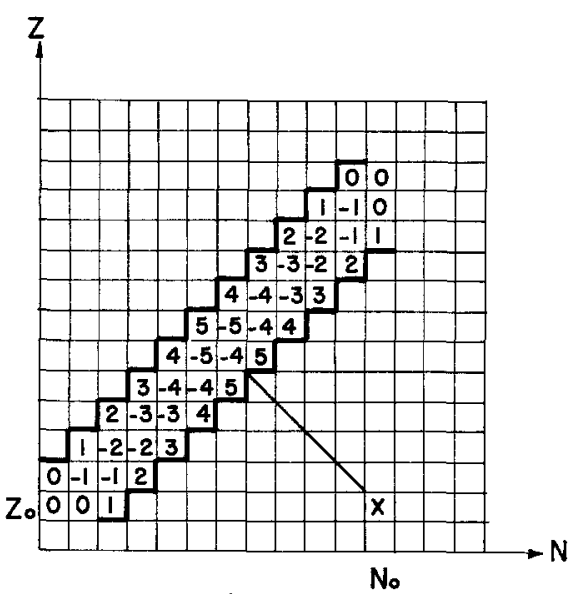

(2)
Fig. 3. A calculation of the mass of a nucleus $\left(Z_{0}, N_{0}\right)$, using the masses of nuclei in a regular skeleton and the transverse mass relations
If nuclei having $A$ and $(A+2)$ nucleons belong to the skeleton, then these nuclei can be denoted thus: $(A, Z) ; \quad(A, Z+1) ; \quad(A+2, Z+1) ; \quad(A+2, Z+2)$ (see Fig. 3 for a description of a regular skeleton).

The regular skeleton will be used to find an expression for the errors of the calculated masses. To this end, let us look at the final table which we shall obtain. This table will satisfy the transverse mass relations. Let us define:

$$
M_{\text {exp }}(Z, N)=M_{\text {calc }}(Z, N)-E(Z, N)
$$

where $E(Z, N)$ is the error of the calculated mass of the $(Z, N)$ nucleus. We assume that $E(Z, N)$ are randomly distributed, having a zero mean and a standard deviation $\sigma_{T}$.

The values of the masses of the nuclei have obviously a physical origin which is not random. It follows that the errors of the mass relations have a physical origin too. The randomness assumption should be taken as a working tool. It serves as a justification for the homogeneous relations, and it is based on the apparent randomness of the errors of the transverse relations. It is obvious that a semiempirical approach to the nuclear mass prediction will always include a random error. If an inhomogeneous term will be accepted and used in mass calculations, then we shall again be left with random errors. In what follows we shall try to analyse the influence of these random errors on the calculated masses.

When we calculate the special mass table from the masses of the nuclei of a certain regular skeleton, we have for every calculated mass an expression which is a linear combination of the masses of the skeleton. This linear combination is unique, since the skeleton is a minimal set. From the equivalence of the expressions (1) and (2) we see, after substituting expression (2) for each mass, that the coefficients defined in Fig. 3 are correct. This expression can easily be generalized for all the nuclei in the range of a regular skeleton. The calculated masses in this special table deviate from the masses of the final table, since in the skeleton of the special table we use the experimental masses, while the final table can be defined if we will substitute the calculated masses of the nuclei in the skeleton.

Using the above assumption about $E(Z, N)$, we have an estimate for the standard deviation of the calculated mass of the nucleus $\left(Z_{0}, N_{0}\right)$ :

$\sigma_{0}^{2} \simeq \sigma_{T}^{2} \cdot \sum_{i} C_{i}^{2}$

where $C_{i}$ are the coefficients displayed in Fig. 3 .

Let us define the quantity $d$ related to that nucleus:

$d=\sum_{i=1}^{n}\left|C_{i}\right|\left(\left|N_{0}-N_{i}\right|+\left|Z_{0}-Z_{i}\right|\right) / \sum_{i=1}^{n}\left|C_{i}\right|$.

In other words, $d$ is the weighted mean distance (in the metric $L_{1}$ ) between the calculated nucleus and the nuclei in the skeleton having a non-zero coefficient. Substituting $d$ in (4) and summing the right hand side, we find an approximate expression for $\sigma_{0}$ for large $d$ :

$\sigma_{0} \cong \sigma_{T} d^{3 / 2} / \sqrt{3}$.

Expression (5) was derived under the assumption that the errors of the transverse mass relations behave as a sample taken from a normal distribution, having a zero mean and a standard deviation $\sigma_{T}$. We turn now to the case where the mean error is not zero. As a special case, let us assume that in the right-hand side of (1) we substitute the constant value $\tau$, and still continue to use the homogeneous relations. In other words, we want to investigate the 


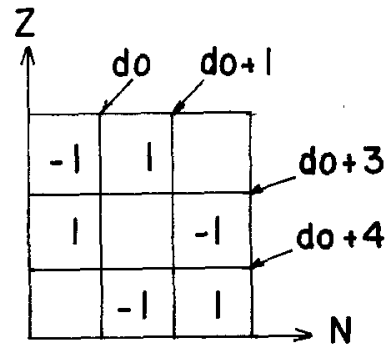

Fig. 4. Scheme of distances of nuclei from the line of beta-stability, as defined and used in the text

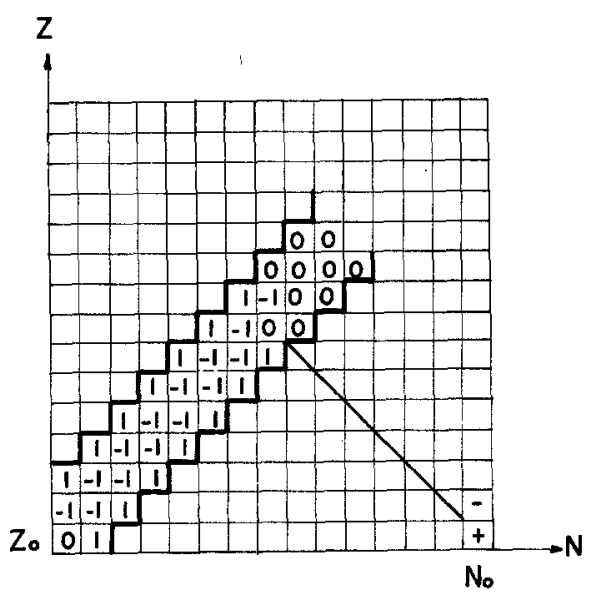

Fig. 5. A calculation of the mass difference of two nuclei, using the mass of nuclei in a regular skeleton and the transverse mass relations

behavior of the errors of the calculated masses, when the homogeneous transverse mass relations have a systematic constant error.

We look at the mass relations of nuclei whose $d$ value varies from $d_{0}$ to $d_{0}+4$ (see Fig. 4 for an illustration). Let us expand the error in powers of $d_{0}$ :

$E\left(d_{0}\right)=\sum_{k} b_{k} d_{0}^{k}$

We substitute this expression in the mass relation of the six nuclei and look at a certain value of $k$. We have

$\sum_{k} b_{k}\left[\left(d_{0}+4\right)^{k}+2\left(d_{0}+1\right)^{k}-2\left(d_{0}+3\right)^{k}-d_{0}^{k}\right]=\tau$.

We see that for every $k$, the coefficients of $d_{0}^{k}, d_{0}^{k-1}$ and $d_{0}^{k-2}$ vanish identically. Since the right hand side is independent of $d_{0}$, we conclude that $b_{k}=0$ for $k>3$, and that $12 b_{3}=\tau$.

It follows that we can write

$E(d)=\tau d^{3} / 12+P(d)$

where $P(d)$ is a second degree polynomial.
We conclude that the propagation of the errors of the calculated masses is much faster in the case where a constant systematic error exists in the homogeneous transverse mass relations, than in the case where only random errors occur in the relations.

The mean deviation of the experimental masses of nuclei having $N, Z \geqq 14$ is about $14 \mathrm{keV}$. A $T$-test of the deviations yields the value $T=0.1$, which indicates that this mean is not significantly different from zero. However, if one uses a mass table derived from the homogeneous transverse mass relations, and if he wants to be cautious and assume that those $14 \mathrm{keV}$ are systematic, then he can use expressions (5) and (7) to see how far can he depart from the line of beta-stability and still not being affected by the assumed constant systematic error of the relations. Using the actual values it is found that for $d \leqq 11$ the random errors are greater than the systematic errors which will emerge from a constant error of $14 \mathrm{keV}$.

We shall try now to find expressions for mass differences. Since we assume that there is no bias in the calculated masses, it is natural to take the difference between the two calculated masses as the value of the mass difference. The situation is not as simple when we look for an expression for the errors related to the calculated mass differences. If the calculations of the masses of the two nuclei were independent, then we would obtain the following expression for the error related to the calculated value:

$\sigma=\sqrt{\sigma_{1}^{2}+\sigma_{2}^{2}}$

We shall show that this is not the case, and that we can predict a much smaller value for the error of the mass difference.

When we look at Fig. 5, we see how the mass difference between two nuclei $\left(Z_{0}, N_{0}\right),\left(Z_{0}+1, N_{0}\right)$ can be expressed as a linear combination of masses of nuclei belonging to a regular skeleton. (In fact, it can be shown that for every skeleton, the respective coefficients will be 1,0 or $(-1)$. Moreover, the coefficients are zero for nuclei in the skeleton having $Z<Z_{0}$ or $A>\left(Z_{0}+N_{0}+1\right)$ in one case, and $Z>\left(Z_{0}\right.$ $+1)$ or $A<\left(Z_{0}+N_{0}\right)$ in another case.)

We see from Fig. 5 that in the case of a regular skeleton, when we substitute the corresponding quantities in (4), we have an estimate for the error of the calculated mass difference of two nuclei $\left(Z_{0}, N_{0}\right)$, $\left(Z_{0}+1, N_{0}\right)$ or $\left(Z_{0}, N_{0}\right),\left(Z_{0}, N_{0}+1\right)$ :

$\sigma_{N}=\sigma_{Z} \cong \sqrt{2} \sigma_{T} \cdot d^{1 / 2} \cong \sqrt{6} \sigma_{0} / d$

where $\sigma_{0}$ is defined in (5). 
Table 1. A statistical $T$-Test of the differences of the errors of two neighboring mass relations along the isospin $T$ direction in the $(Z, N)$ plane. It is seen that there is no significant difference between this quantity and zero

\begin{tabular}{lcccccc}
\hline Mass relation & Region & Mean & S.D. & $\begin{array}{l}T \\
\text { statistic }\end{array}$ & $\begin{array}{l}\text { Degrees } \\
\text { of freedom }\end{array}$ & $\begin{array}{l}\text { Signifi- } \\
\text { cance }\end{array}$ \\
\hline Transverse & $A \geqq 28$ & -22.2 & 305.6 & -1.18 & 260 & 0.24 \\
& $80 \geqq A \geqq 28$ & -35.0 & 371.2 & -0.72 & 57 & 0.48 \\
& $160 \geqq A>80$ & -32.7 & 293.0 & -1.26 & 126 & 0.21 \\
& $A>160$ & -5.0 & 270.6 & 0.16 & 75 & 0.87 \\
Longitudinal & $A \geqq 28$ & +3.4 & 283.6 & 0.24 & 372 & 0.81 \\
& $80 \geqq A \geqq 28$ & +19.7 & 341.4 & 0.57 & 96 & 0.57 \\
& $160 \geqq A>80$ & -3.6 & 286.8 & -0.16 & 161 & 0.81 \\
& $A>160$ & -0.4 & 219.5 & -0.02 & 113 & 0.99 \\
\hline
\end{tabular}

In a similar way we can find the following expressions for the errors related to the calculated mass differences of the following pairs of nuclei $\left(Z_{0}, N_{0}\right),\left(Z_{0}-1, N_{0}+1\right)$ and $\left(Z_{0}, N_{0}\right),\left(Z_{0}+2, N_{0}+2\right)$.

$\sigma_{\beta} \cong \sqrt{2} \sigma_{N} \cong 2 \sigma_{T} \cdot d^{1 / 2} \cong \sqrt{12} \sigma_{0} / d$

$\sigma_{\alpha} \cong 2 \sigma_{\beta} \cong \sqrt{8} \sigma_{N} \cong 4 \sigma_{T} d^{1 / 2}=4 \cdot \sqrt{3} \cdot \sigma_{0} / d$.

Expression (5) was compared with the values of the errors, as obtained in the actual calculation [1]. We note that this comparison is done even though the skeletons in [1] were irregular and expression (5) is an asymptotic limit of a better expression.

The quantity $d_{i}$ defined above for the $i^{\prime}$ th nucleus, is a function of $Z_{i}, N_{i}$ and of the specific skeleton. We represent the set of quantities $d_{i}$, which varies from skeleton to skeleton with the quantity $D_{i}$ which is the distance of the $i$ th nucleus from the line of betastability. This value can be taken as a fair representation of the mean of all the $d_{i}$.

Using the actual values of the errors and the quantity $D_{i}$, we tried to fit the two parameters $\alpha$ and $\beta$ in the following expression:

$\sigma_{i}=\alpha D_{i}^{\beta}$.

The values obtained from the fit are:

$\alpha=42 \pm 2.5 \mathrm{KeV}$

$\beta=1.55 \pm 0.05$.

We see that the value of $\beta$ is in accordance with expression (5). The value of $\alpha$ is lower than expected. The root mean square of the error of the calculated masses of the nuclei whose masses are known experimentally was $168 \mathrm{KeV}$. Thus, the value of $\alpha$ should be $168 / \sqrt{3} \cong 100 \mathrm{KeV}$. We relate this discrepancy to the various approximations done during the derivation of expression (5) and in its adaptation to the actual data.
From the above analysis we can derive some conclusions about the pattern of the calculated errors. We saw that the calculated errors of the masses of nuclei far from the line of beta-stability behave as $d^{3 / 2}$, while the errors of mass differences behave as $d^{1 / 2}$. Thus, if a calculated mass of a nucleus whose location is far from the line of beta-stability, has a typical error $+E$, it is reasonable that its neighbors will have a positive error, too. From here we find that the greater the distance from the line of betastability, the larger will be the "islands" of error of the same sign.

We conclude this work with a few lines about the possible systematic error of the mass relations, when tested far from the line of beta stability. A serious answer to this problem will be found either when we have a well-established theory of nuclear interactions or when these masses are measured experimentally. At present, we are far from both solutions. In what follows, we describe a test from which we find that at least we have no indication of a systematic error in mass relations, when applied to nuclei far from the line of beta-stability.

We want to find an indication concerning the variation of the errors of the mass relations, when we depart from the line of beta stability. To this end, we define two curvilinear coordinates $(\alpha, \tau)$, where $\alpha_{0}$ is a smooth curve along the line of beta-stability, and is perpendicular to $\tau$. Let us define $\tau=0$ for the line $\alpha$ along the line of beta stability, and look at the formal expansion of the error of the mass relations in terms of $\alpha, \tau$, and retain the linear terms only:

$R(\alpha, \tau)=R_{0}+a \alpha+t \tau$.

We know from the data that $R_{0}$ and $a$ have no significant difference from zero. On the other hand, we know the errors only near the origin of the $\tau$ coordinate. To find some information about $t$, we return to the $T$ direction, which produces a small 
angle $\theta$ with respect to $\tau$, and thus $\cos (\theta)$ is not far from unity. We define:

$V(N, Z)=R(N, Z)-R(N-1, Z+1)$.

A statistical $t$-test was performed in order to find if the quantity $V(N, Z)$ is significantly different from zero. It has been found (see Table 1) that there is no significant difference between the quantity $V(N, Z)$ and zero. This was found for both the transverse and longitudinal relations, and also when applied separately to regions of low, medium and high $A$.

This $T$-test shows that if the existence of an inhomogeneous term will be established, then it will not take a simple form, since it is statistically reasonable that its linear expansion in terms of $A$ and $\tau$ will have coefficients not far from zero.

From this we derive that when we calculate masses of nuclei far from the line of beta stability, using the transverse mass relations and the experimental masses as an extrapolative base, we have no statistical confirmation of systematic errors, but of random er- rors only. The behavior of the random errors was described above.

\section{References}

1. Comay, E., Kelson, I.: At. Data Nucl. Data Tables 17, 463 (1976)

2. Garvey, G.T., Gerace, W.J., Jaffe, R.L., Talmi, I., Kelson, I.: Rev. Mod. Phys. 41, S1 (1969)

3. Janecke, J.: At. Data Nucl. Data Tables 17, 455 (1976)

4. At. Data Nucl. Data Tables 17, 476 (1976)

E. Comay

Department of Physics

David M. Dennison Building

University of Michigan

Ann Arbor, MI 48109

USA

I. Kelson

Department of Physics and Astronomy

Tel Aviv University

Tel Aviv, 69978

Istael 